\title{
Corela
}

Cognition, représentation, langage

HS-13 | 2013

Statut et utilisation des corpus en linguistique

\section{Corpus et alternance codique : que peut nous apprendre une approche comparative?}

\section{Charles Brasart}

\section{OpenEdition}

\section{Journals}

Édition électronique

URL : http://journals.openedition.org/corela/3042

DOI : $10.4000 /$ corela.3042

ISSN : $1638-573 \mathrm{X}$

Éditeur

Cercle linguistique du Centre et de I'Ouest - CerLICO

Référence électronique

Charles Brasart, «Corpus et alternance codique : que peut nous apprendre une approche comparative ?», Corela [En ligne], HS-13 | 2013, mis en ligne le 10 décembre 2013, consulté le 19 avril 2019. URL : http://journals.openedition.org/corela/3042 ; DOI : 10.4000/corela.3042

Ce document a été généré automatiquement le 19 avril 2019

\section{(c) (i) (2)(2)}

Corela - cognition, représentation, langage est mis à disposition selon les termes de la licence Creative Commons Attribution - Pas d'Utilisation Commerciale - Partage dans les Mêmes Conditions 4.0 International. 


\title{
Corpus et alternance codique : que peut nous apprendre une approche comparative?
}

\author{
Charles Brasart
}

The work of the last thirty years has produced an
impressive corpus of bilingual speech illustrating
code switching and a host of other language
interaction phenomena. Clearly the field owes
whatever achievements have been attained to this
technique.
Gullberg, Indefrey et Muysken (2009:23)

\section{Introduction}

1 La recherche sur corpus a acquis une place de plus en plus importante en linguistique au cours des dernières années, comme peut le montrer la citation de Gullberg, Indefrey et Muysken que nous avons placée en épigraphe de cet article. Elle est cependant une méthodologie parmi d'autres, et ne fait pas nécessairement l'unanimité. Dans le champ de l'étude de l'alternance codique, cet outil fait concurrence aux jugements intuitifs d'acceptabilité, émis par des locuteurs natifs sur des énoncés qui leur sont présentés par les chercheurs, et il nous paraît judicieux de poser la question du statut et du crédit à accorder à chacune de ces deux méthodologies. La première est un outil inductif, empiriste, qui permet de partir de l'observation de données pour en déduire des modèles théoriques, tandis que l'autre est un outil hypothético-déductif, rationaliste, qui permet de mettre des systèmes théoriques à l'épreuve des intuitions grammaticales de locuteurs. Ce sont donc deux méthodologies aux fonctionnements diamétralement opposés et qui font l'objet de débats féroces au sein de la communauté qui les utilise, tout comme 
d'ailleurs la définition exacte de ce qu'est l'alternance codique. Poplack, l'une des grandes spécialistes du sujet, écrit ainsi:

"Code-switching is but one of a number of the linguistic manifestations of language contact and mixing, which variously include borrowing on the lexical and syntactic levels, language transfer, linguistic convergence, interference, language attrition, language death, pidginization and creolization, among others. There is little consensus in the literature over which aspects should be subsumed under the label code-switching." (2004:1)

Gardner-Chloros, autre autorité sur la question, en donne une définition fort similaire :

"Code-switching: This is a general term which refers to the alternate use of two or more languages or language varieties by bilinguals for communicative purposes. Code-switching embraces various types of bilingual behavior such as switching within and between utterances, turns and sentences." (2009: 202)

On notera que Poplack fait une différence entre code-switching et code-mixing. Ce second terme est utilisé dans notre champ depuis 1986, date de parution d'un article de Di Sciullo, Muysken \& Singh dans lequel les auteurs font la distinction entre alternance, phénomène dans lequel les deux langues restent séparées, et mélange, phénomène dans lequel des éléments lexicaux et des traits grammaticaux des deux langues apparaissent dans la même phrase. Pour Pfaff (1979), cependant, le terme d'alternance codique doit être pris comme un hyponyme de mélange codique, phénomène qui inclut selon elle également les emprunts. La question de savoir quel sens et quelle place accorder aux deux termes et de déterminer quelles relations ils entretiennent l'un par rapport à l'autre fait tellement débat que Gardner-Chloros va jusqu'à proposer de ne plus les utiliser et de leur préférer l'expression de "language interaction » (2009: 202). Nous préférons quant à nous conserver le terme d'alternance codique, qui est canonique, tout en précisant la définition que nous en faisons. L'acception que nous prêtons au terme, qui est volontairement large, est inspirée de définitions que l'on trouve chez Gumperz :

"[Code-switching is the] juxtaposition within the same speech exchange of passages of speech belonging to two different grammatical systems or subsystems." (1982:

59)

ainsi que chez Bullock \& Toribio:

"[Code-switching is] the alternating use of two languages in the same stretch of discourse by a bilingual speaker." (2009 : xii)

et:

"Code-switching is the ability on the part of bilinguals to alternate effortlessly between their two languages." (2009: 1)

ou encore chez Gardner-Chloros:

"[Code-switching] refers to the use of several languages or dialects in the same conversation or sentence by bilingual people." (2009:4)

Notre propre définition du phénomène sera donc la suivante :

L'alternance codique est l'usage fluide de deux langues ou plus au cours de la même conversation par un ou plusieurs locuteurs bilingues.

Cela signifie donc que nous incluons dans l'alternance codique les cas où un locuteur parle une langue A, par exemple le français, et un autre une langue B, par exemple l'anglais. En effet, même si ces deux locuteurs ne parlent chacun qu'une seule langue, ils n'en utilisent pas moins les deux, puisqu'ils écoutent, comprennent et réagissent à la langue de l'autre. Ils se servent donc bien, de façon fluide et au cours de la même conversation, de deux langues. 
5 La recherche sur le mélange des langues dans la conversation bilingue a longtemps reposé sur l'analyse d'énoncés inventés par les linguistes, et sur des jugements intuitifs d'acceptabilité (MacSwan, 1999). Nous inscrivant dans une tendance plus récente reposant sur l'étude de faits de langue qui ne sont plus pris isolément mais replacés dans leur contexte de production, nous souhaitons démontrer que la recherche sur corpus est un outil indispensable pour la compréhension des phénomènes d'alternance codique chez les locuteurs bilingues, bien qu'étant insuffisant pour produire un appareil théorique infaillible. En effet, elle présente par essence des limitations que seules des approches complémentaires semblent à même de dépasser afin de produire des résultats qui ne soient pas applicables qu'au seul corpus envisagé. Nous tenterons ainsi de montrer qu'une approche fondée sur la comparaison de deux corpus peut permettre de dégager des généralisations qui ne s'appliquent pas à la seule sphère étudiée. Nous comparerons en premier lieu les deux méthodologies traditionnellement employées dans notre champ, à savoir étude sur corpus et jugements d'acceptabilité. Puis nous présenterons notre propre méthodologie. Enfin, nous discuterons des généralisations qu'il est possible ou non d'en tirer, en nous penchant sur des exemples de phénomènes communs aux deux corpus et sur les questions qui restent en suspens.

\section{Jugements d'acceptabilité et corpus}

Dès les premiers pas de la recherche sur l'alternance codique, en lien avec l'émergence des théories générativistes, ce sont les considérations grammaticales qui ont constitué le fer de lance de la recherche sur le sujet, les linguistes cherchant à établir des règles universelles qui s'appliqueraient indépendamment des locuteurs et des langues. Les analyses étaient alors fondées en grande partie sur des jugements d'acceptabilité. Or, plus encore que lorsqu'une seule langue est en jeu, dans le cadre du mélange de deux langues, sur quelles règles grammaticales peut-on s'appuyer pour justifier rejet ou acceptation? MacSwan, par exemple, déclare la phrase (2) ci-dessous inacceptable et pose la question suivante :

(1) Mi hermano bought some ice cream.

(2) *El bought some ice cream.

"Why would a bilingual's grammar permit switching between a lexical DP and verb

but not between a pronoun and verb ?" $(2010: 1)$

Or on trouve facilement des contre-exemples qui montrent que les locuteurs peuvent changer de langue entre un pronom sujet et un verbe :

(a) Et donc du coup toi i'faut vraiment qu'tu stumble out, quoi, vraiment.

(b) Na ja, ich enjoy's, aber ich muss mich so... konzentrieren.

Le jugement d'acceptabilité, qui exclut du champ des possibles certaines combinaisons de langues que l'on trouve dans les corpus de données naturelles, semble donc être un outil périlleux à manier, et ce d'autant plus dans le domaine de l'alternance codique, qui est une pratique souvent connotée négativement. Dès lors, on constate fréquemment un écart entre ce que font les locuteurs et ce qu'ils disent qu'ils font.

9 Si l'alternance codique doit affronter de telles attitudes négatives, c'est parce qu'elle s'écarte intrinsèquement de ce qui est perçu comme la norme, c'est-à-dire le monolinguisme. L'alternance codique est une sorte d'entre-deux qui, ayant un pied dans chaque langue, n'est totalement ni dans l'une ni dans l'autre et ne peut donc respecter les règles édictées par les grammaires. C'est un phénomène qui crée ses propres règles au fur 
et à mesure qu'il apparaît dans chaque groupe de locuteurs bilingues. Il n'est pas enseigné à l'école, il n'est codifié par personne, et en cas de doute il n'est pas possible d'aller chercher le Grevisse de l'Alternance Codique dans sa bibliothèque. Mais c'est à ce niveau précis qu'apparaît le problème fondamental que pose l'alternance codique quant à l'usage des jugements de grammaticalité : s'il est indéniable que c'est un phénomène grammatical (puisqu'on n'y utilise pas les mots au hasard), ce n'est pas un phénomène qui relève des grammaires. Le jugement d'acceptabilité repose sur l'idée que certaines tournures sont correctes, d'autres non ; or l'alternance codique échappe complètement à ce carcan. Et si un corpus peut parfaitement saisir cela, les jugements d'acceptabilité, par leur nature même, en sont incapables, car ils participent de l'extrême inverse de la spontanéité linguistique et reposent sur une conception de la langue en tant que liste de règles syntaxiques apprises, plutôt que comme une intériorisation des outils disponibles pour véhiculer du sens. Or, comme l'écrit Gardner-Chloros:

"The outcome of specifying a "grammar" of $\mathrm{CS}^{1}$ is that there appears to be a right and a wrong way to code-switch, or at least a "possible" and an "impossible" way." (2009: 92)

Un deuxième problème posé par les énoncés fabriqués pour les jugements d'acceptabilité est qu'ils ressemblent souvent à des phrases que l'on trouverait dans des grammaires, prononcées par un locuteur idéal, plutôt qu'à des productions linguistiques naturelles. Or la langue parlée fonctionne fréquemment avec hésitations, répétitions, faux départs, etc. C'est ce que l'on peut voir dans un exemple comme le suivant :

(c) PIE : Au moment où tu l'as fait c'était très bien; seulement, à c'moment, sur cette phrase-là par exemple, « oh relief, machin ", pense, toi, peut-être, que, pense à c'moment-là, que, y'a, genre, en fait euh, un truc euh... genre un gode dans l'chest et c'est la honte en fait [Rires] et que, LAU : $\{[$ Rire $]$ Un énorme... grand comme ça...

PIE : \{tu voudrais pas qu'il le voie et donc tu vas voir Granno tu vas lui dire : «Oh chuis relieved! I thought you'd find, you'd find the real truth ", genre un truc dont, pour l'quel c'est la honte!'

11 On note immédiatement que la syntaxe de ces énoncés, sans même encore parler d'alternance codique, est loin d'être canonique : répétitions (" pense, toi, peut-être, que, pense à c'moment-là, que »), hésitations ("y'a, genre, en fait euh, un truc euh»), dislocations ("genre un truc dont, pour l'quel c'est la honte!») en font des énoncés typiques de l'oral. S'ils étaient soumis à un jugement d'acceptabilité de locuteurs natifs, il fait peu de doute que le verdict serait unanime quant à leur caractère incorrect. Mais cela ne changerait rien au fait que ces énoncés ont été prononcés par un énonciateur natif, et compris par d'autres. Partant, ce que la recherche sur corpus peut permettre de faire, c'est étudier comment les locuteurs dévient de la norme grammaticale dans les productions spontanées, ce que les jugements d'acceptabilité ne permettent pas de faire.

12 L'analyse sur corpus présente ainsi l'avantage de pouvoir mêler approche grammaticale, analyse du texte et approche sociolinguistique : elle livre les énoncés dans leur contexte de production et permet d'observer la langue telle qu'elle se construit à force d'échanges au sein d'un groupe donné. De plus, elle rend possible une analyse statistique des faits observés, permettant ainsi de déterminer quels sont les phénomènes récurrents et quels sont ceux qui sont exceptionnels. Ce type d'approche permet donc une analyse de la pratique de la langue. Cela, cependant, implique de se poser la question suivante: si l'alternance codique est une pratique construite par l'individu au sein du groupe, phénomène de surcroît marginal dans les sociétés monolingues, est-il possible de 
généraliser les faits observés et les conclusions qui en sont tirées ? Comment s'assurer que les faits de langue observés ne sont pas dus aux idiosyncrasies du groupe par lesquels ils ont été produits? Une comparaison entre deux corpus peut permettre d'apporter des éléments de réponse.

\section{L'analyse de corpus croisée}

13 Une étude de corpus croisée, dans laquelle sont comparés les phénomènes relevés chez deux groupes de locuteurs, peut permettre de dégager quelles sont les tendances générales (si elles existent) qui émergent lorsqu'un groupe de locuteurs utilise deux langues pour converser, indépendamment dudit groupe. Saussure parle au sujet de la parole d'« associations ratifiées par le consentement collectif » (1913:32); or il ne peut y avoir de consentement entre deux groupes séparés de façon étanche, que ce soit par le temps, l'espace, la langue ou encore l'âge. Les éventuelles récurrences d'un corpus à l'autre seront donc imputables à la compétence des locuteurs bilingues et susceptibles d'être déclarées universelles, ou tout du moins générales.

Dans le cadre de cette étude, nous avons utilisé deux corpus. Le premier est un corpus de conversation français-anglais s'étendant sur environ une quinzaine d'heures d'enregistrement audio. L'enregistrement s'est fait à l'occasion de répétitions hebdomadaires de théâtre, de 2009 à 2011, ce qui permet d'avoir un échantillon de productions linguistiques échelonnées dans le temps plutôt que toutes recueillies dans la même situation d'énonciation. Cela est un moyen de nous assurer que ces productions ne sont pas un avatar de l'enregistrement mais représentent une façon naturelle de s'exprimer chez les informants. Ceux-ci sont au nombre de neuf, cinq femmes et quatre hommes, âgés de vingt-cinq à trente-deux ans, tous bilingues.

Le second corpus sur lequel nous fondons nos analyses est un corpus de conversation anglais-allemand, compilé par Eppler $(1993)^{3}$ et obtenu grâce au projet TalkBank. Comme dans le cas précédent, ce corpus a été enregistré auprès de neuf personnes, sept femmes et deux hommes, et s'étend sur une douzaine d'heures. Le choix de ce corpus a été en partie motivé par les deux langues qui y sont parlées : le fait que l'anglais soit une langue commune aux deux corpus, et que parmi les deux autres langues, l'une soit germanique, l'autre romane, peut prêter à de nombreuses comparaisons en fonction des couples de langues et de leurs idiosyncrasies syntaxiques respectives. Autre critère important: les informants de ce corpus appartiennent à une catégorie socioprofessionnelle radicalement différente des nôtres. Tous sont des personnes âgées (déjà à l'époque à laquelle a été recueilli le corpus), qui ont fui l'Autriche en 1938 et se sont installés à Londres. Les situations sont donc foncièrement différentes entre les deux corpus, et ces oppositions sont selon nous importantes pour la raison suivante: elles peuvent potentiellement permettre de séparer les phénomènes linguistiques rencontrés dans chaque corpus de leurs conditions de production. De la sorte, il devient théoriquement possible de déterminer si lesdits phénomènes sont intimement et irrémédiablement liés aux locuteurs qui les produisent, ou bien, au contraire, si l'usage de deux langues dans la même prise de parole mène à l'émergence de phénomènes similaires indépendamment des locuteurs et des langues.

Le corpus anglais-allemand contient à peu près 95000 mots, et 1500 énoncés bilingues environ. Notre corpus, par comparaison, contient 50000 mots, soit 1,9 fois moins, et 811 énoncés bilingues, ce qui représente à très peu de choses près le même ratio, c'est-à-dire 
1,85. Ainsi, dans le corpus anglais-allemand, il se produit statistiquement un changement de langue tous les 63 mots, et dans le nôtre, tous les 61 mots. Il semblerait donc que, au moins sur le plan statistique, nos informants et ceux d'Eva Eppler alternent les langues à la même fréquence. Mais, en premier lieu, l'intérêt est de pouvoir, pour chaque corpus, voir sous quelles formes se manifestent les phénomènes d'alternance codique, dont on peut voir la répartition dans les figures ci-dessous.

Figure 1 : Répartition des phénomènes dans le corpus Français-Anglais

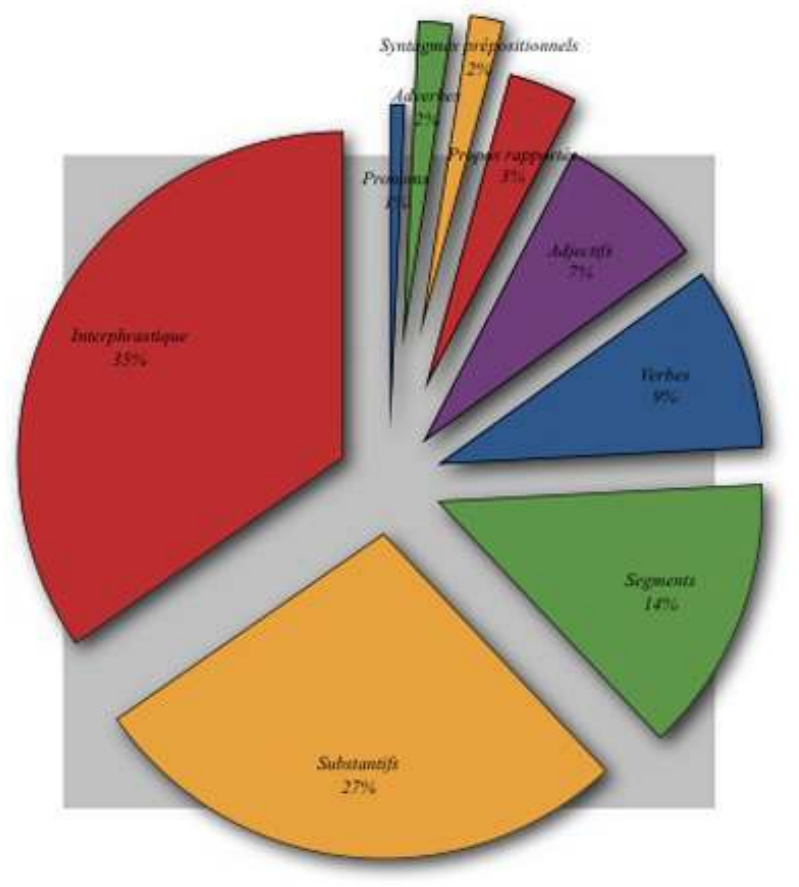


Figure 2 : Répartition des phénomènes dans le corpus Allemand-Anglais

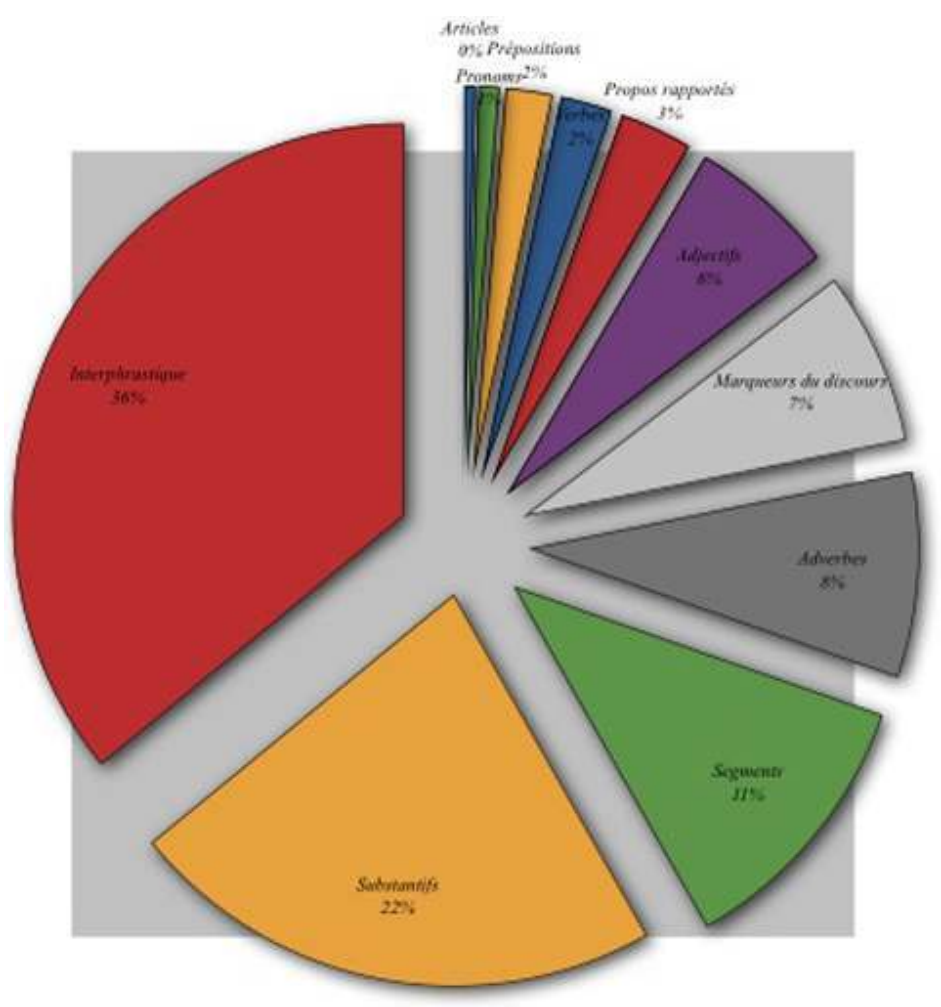

On observe que les trois catégories les plus représentées sont les mêmes dans l'un et l'autre corpus: les manifestations d'alternance interphrastique, où le changement de langue se fait entre deux phrases; l'« emprunt » de substantifs, et les segments en langue $B$ insérés dans un énoncé en langue A, par exemple des constructions idiomatiques ou des syntagmes. Ce qui frappe d'emblée, c'est que les proportions dans lesquelles ces trois phénomènes apparaissent sont presque identiques dans les deux corpus: $34,65 \%$ et $35,91 \%$ pour la première catégorie, soit un tiers des phénomènes, $27,37 \%$ et $22,33 \%$ pour les substantifs, soit un quart, et 13,81 et $11,43 \%$ pour les segments. Dans l'un et l'autre corpus, ces trois catégories représentent respectivement $75,83 \%$ et $69,67 \%$ des énoncés bilingues, soit près des trois quarts du total. On notera ensuite que la cinquième catégorie la plus représentée dans l'un et l'autre corpus, les adjectifs, fait l'objet de 7,40\% des mélanges dans le corpus français-anglais et de $6,19 \%$ du total dans le corpus anglaisallemand, soit des proportions à nouveau relativement similaires. Enfin, les catégories moins représentées le sont également dans des proportions très proches dans les deux corpus : $3,45 \%$ et $3,03 \%$ pour les propos rapportés, $1,73 \%$ et $1,95 \%$ pour les prépositions et syntagmes prépositionnels ${ }^{4}, 0,74 \%$ et $0,87 \%$ pour les pronoms. Les catégories concernées ont ainsi une distribution extrêmement similaire dans l'un et l'autre groupe de locuteurs.

Nos deux corpus présentent donc une répartition dont les similitudes ont de quoi surprendre. Cette répartition montre que l'alternance codique semble se faire selon les mêmes modalités indépendamment du groupe, en dépit de caractéristiques propres à l'un ou l'autre corpus mais qui sont relativement marginales en termes de quantité. 


\section{Points communs et différences}

$19 \mathrm{Au}$ regard de leur fréquence d'apparition, on peut en premier lieu se pencher sur le nombre de cas de substantifs de langue B utilisés dans un énoncé en langue A dans chaque corpus, comme dans les exemples suivants :

(d) DOR : Die Fritzi ist mit der Spitz gefahren, mit der Stein gefahren, voriges Jahr zu Weihnachten, nach Mallorca, glaube ich.

LIL : Ja, sie wollten das Taxi am airport.

DOR : Wollten sie, hat die Fritzi gesagt... Und wenn die Fritzi schon sagt, sie fahren zusammen, nehmen sie ein Taxi heraus... because das war Christmas Eve, wie sie zurückkommen, hat sie gesagt, sie hat die travelcard, sie fahrt nicht. ${ }^{5}$

(e) LAU : [...] toi tu viens, tu veux du, tu veux du rope, c'est la suite de ça, hein...

PIE : Ah, ça c'est la suite, d'accord.

LAU : Tu veux du rope... blablabla..

CAR : Et après eux ils arrivent.

LAU : Et eux i'viennent, i'cherchent le rope, i's'mettent à faire des parcels, sur le trunk, c'est ça?

Nous relevons 177 occurrences de ce cas dans le corpus français-anglais, soit $21,82 \%$ des cas d'alternance codique. Par comparaison, nous en trouvons 262 dans le corpus anglaisallemand, soit $17,62 \%$ des phénomènes observés. Leur fréquence d'apparition est donc sensiblement similaire dans l'un et l'autre corpus. Si l'on inclut dans le décompte non seulement les noms mais également les syntagmes nominaux, les totaux montent à respectivement $24,41 \%$ et $22,33 \%$, la différence devenant encore plus ténue. Il est intéressant de constater qu'ils représentent entre le cinquième et le quart des phénomènes observés dans nos deux corpus, et l'on peut se demander si cette similitude est révélatrice d'une tendance plus générale dans l'alternance codique ou la langue. On pourrait ainsi penser que cette proportion est le reflet de la proportion de noms communément employés dans toute prise de parole, mais, d'après nos statistiques, les substantifs représentent moins de $10 \%$ des mots de nos corpus. De plus, il serait alors logique que les autres grandes catégories lexicales soient représentées de la même façon dans nos deux corpus. Or, si l'on prend les verbes, par exemple, qui sont une autre catégorie incontournable dans la construction d'énoncés grammaticaux, les proportions ne sont plus du tout les mêmes. Ceux-ci représentent en effet 9,12 \% des manifestations d'alternance codique dans le corpus français-anglais, alors qu'ils ne concernent que $2,15 \%$ des phénomènes dans le corpus anglais-allemand, c'est-à-dire quatre fois moins, ce qui est fort peu en termes relatifs aussi bien qu'absolus. À l'inverse, les adverbes concernent $8,47 \%$ des cas dans le corpus anglais-allemand mais seulement $1,47 \%$ des cas dans l'autre corpus.

21 Si l'on se penche sur la fonction, lorsque cela est possible, d'autres éléments de réflexion viennent se superposer au tableau peint par la répartition par natures. Ainsi, 9,37\% des occurrences d'alternance dans le corpus anglais-français et 6,19\% d'entre elles dans le second corpus remplissent la fonction d'attribut du sujet, tandis que respectivement $3,95 \%$ et $4,03 \%$ des occurrences remplissent la fonction de complément d'objet du verbe, pour prendre deux fonctions se trouvant typiquement en position finale dans la phrase. La répartition est donc relativement similaire. Si l'on considère la grande diversité des possibilités d'alternance, il peut être frappant de constater que ces deux fonctions représentent entre 10 et $13 \%$ des phénomènes, d'autant que l'on pourrait s'attendre, notamment dans le premier cas, à ce que le sujet et son attribut soient dans la même 
langue. Le second attribuant une qualité au premier, en effet, on pourrait croire qu'il serait plus simple qu'ils appartiennent tous deux au même plan de réalité. Or une même langue semble un premier pas logique vers une relation d'identité. De même, séparer par la langue le processus et son objet peut donner l'impression qu'il émerge une cassure dans le scénario verbal. Mais cela apparaît contre-intuitif dès lors que l'on se défait de la vision fractionnelle du bilinguisme dont parle Grosjean (2008) : si le bilinguisme peut être vu en partie comme une capacité à se définir de façon duelle, le changement de langue n'implique pas nécessairement une séparation entre les éléments phrastiques. Au contraire, il assure cohérence et cohésion par le recours aux deux filtres à travers lesquels le bilingue découpe la réalité.

Deuxièmement, l'on ne devrait pas être étonné de ce que les places d'attribut du sujet et d'objet du verbe soient si fréquemment instanciées par des éléments de langue $B$, dans la mesure où ce sont des positions focalisantes, même quand elles ne sont pas finales, qui véhiculent l'information nouvelle de la prise de parole. Le fait de passer à une langue $\mathrm{B}$ au moment d'instancier ce créneau lexical permet dès lors, par le truchement du changement, de faire en sorte de créer un énoncé assez frappant pour que l'élément important soit d'autant plus saillant et donc remarquable, au sens premier du terme. C'est typiquement ce que l'on trouve selon nous dans un exemple comme le suivant, où le passage à l'anglais pour l'attribut du sujet rythme la phrase et s'en détache tel un élément surligné :

(f) Wenn du short bist, you wouldn't talk. Aber wenn man Geld hat, you talk. ${ }^{6}$

On remarquera d'ailleurs que le même processus est à l'œuvre entre protase et apodose dans les deux phrases de l'énoncé, le changement de langue permettant d'accompagner le passage de la condition à la conséquence. C'est ce que l'on trouve également dans un énoncé aussi simple que le suivant :

(g) SOP : Attention là c'est heavy!

Le fait de qualifier ce dont il est question (un carton de livres) en anglais à la fin d'une structure en français permet d'attirer d'autant plus l'attention du destinataire sur ce à quoi il convient, justement, de faire attention.

\section{Conclusion}

Comme le montrent nos statistiques, l'analyse croisée permet de dégager à la fois ce qui semble être des tendances se retrouvant dans les deux groupes étudiés et des pratiques propres à chacun. Cela tend à prouver qu'il existe des modalités d'alternance générales, indépendantes des locuteurs étudiés. Le changement de langue en fin d'énoncé, ou entre deux phrases, l'emprunt de substantifs à la langue $B$ sont des phénomènes très présents et aux fréquences d'apparition comparables dans les corpus, signe probable qu'il s'agit d'une tendance plus globale. Mais notre étude montre également qu'il peut être dangereux de considérer l'alternance codique en tant que phénomène en soi, détaché de tout ancrage discursif. Il conviendrait selon nous de l'envisager comme une pratique menant à l'émergence de sociolectes bien particuliers, dans lesquels certains des faits observés sont la conséquence de la dynamique du groupe. Il est dès lors indispensable d'intégrer les locuteurs comme facteur dans l'analyse du phénomène et de se poser la question de l'échelle à laquelle les conclusions tirées peuvent s'appliquer: l'on peut parler de tendances générales, mais il serait probablement vain de tenter de dégager des universaux, d'autant plus qu'il peut y avoir autant de pratiques que de groupes de 
locuteurs. L'étude de corpus devient alors indispensable à une compréhension plus fine du phénomène, et si elle ne permet pas de parvenir à des règles pouvant prédire quelles formes peut prendre ou non le mélange des codes, elle n'en est pas moins l'outil le plus apte à décrire ces formes de façon correcte et, partant, à fonder une analyse sur des bases épistémologiques saines.

\section{BIBLIOGRAPHIE}

Bullock, Barbara \& ToRiBio Almeida Jacqueline (dir.) (2009). The Cambridge Handbook of CodeSwitching. Cambridge University Press: Cambridge.

DI SCIULlO, Anne-Marie, MUYSKEn Pieter \& SINGH Rajandra (1986). "Government and codeswitching". Journal of Linguistics, 22 (1). 1-24.

EPPLER, Eva (1993). "German/English LIDES Data, an approx. 95,000 word LIDES/CHAT transcribed corpus of German/English bilingual speech with audio links to digitized files of the original audio recordings". http://talkbank.org/data/LIDES/Eppler.zip, ISBN 1-59642-218-1.

GARDNER-CHLOROS, Penelope (2009). Code-Switching. Cambridge: Cambridge University Press. GrosjeAN, François (2008). Studying Bilinguals. Oxford: Oxford University Press.

GUllberg, Marianne, InDefRey Peter \& MuYsKen Pieter (2009). "Research techniques for the study of code-switching". In The Cambridge Handbook of Linguistic Code-Switching, Bullock Barbara \& Toribio Almeida Jacqueline (dir.). Cambridge: Cambridge University Press. 20-39.

GUMPERZ, John (1982). Discourse strategies. Cambridge: Cambridge University Press.

LABOV, William (1972). Sociolinguistic Patterns. Philadelphia: University of Pennsylvania Press.

MacSwAN, Jeff (1999). A Minimalist Approach to Intrasentential Code Switching. New York: Garland Press.

MacSwAN, Jeff (2010). "Plenary address: Unconstraining codeswitching theories". Proceedings from the Annual Meeting of the Chicago Linguistic Society 44. Chicago: University of Chicago Press.

PFAFF, Carol (1979). "Constraints on language mixing". Language, 55. 291-318.

POPLACK, Shana (2004). "Code-switching". In Soziolinguistik. An international handbook of the science of language. Ammon Ulrich, Dittmar Norbert, Mattheier Klaus J. \& Trudgill Peter (dir.). Berlin : Walter de Gruyter.

ROMAINE, Suzanne (1995). Bilingualism. Oxford : Blackwell.

SAUSSURE, Ferdinand de, 1967 [1913]. Cours de linguistique générale. Wiesbaden: Otto Harrassowitz.

\section{NOTES}

1. CS : code-switching

2. Les énonciateurs discutent de leurs rôles dans une pièce de théâtre en anglais. 
3. Eppler, Eva, German/English LIDES Data (An approx. 95,000 word LIDES/CHAT transcribed corpus of German/English bilingual speech with audio links to digitized files of the original audio recordings), http://talkbank.org/data/LIDES/Eppler.zip, 1993.

4. On notera cependant qu'il est fréquent que seules les prépositions fassent l'objet d'alternance dans le corpus anglais-allemand alors qu'il est beaucoup plus fréquent dans l'autre corpus que ce soient des syntagmes qui soient concernés.

5. « Fritzi est partie avec Spitz, avec Stein, il y a quelques années, pour Noël, à Majorque, je crois. - Oui, ils voulaient prendre un taxi à l'aéroport. - Ils voulaient, a dit Fritzi... Et quand Fritzi a dit qu'ils voyageraient ensemble, qu'ils partiraient en taxi... parce que c'était la veille de Noël, quand ils sont revenus, elle a dit qu'elle avait une travelcard (abonnement pour les transports en commun), elle ne prend pas de taxi.

6. «Quand tu n'as pas assez, tu ne parles pas. Mais quand tu as de l'argent, tu parles.»

\section{RÉSUMÉS}

Cet article a pour but de démontrer que la recherche sur corpus est le moyen le plus adapté pour décrire correctement les phénomènes d'alternance codique. En replaçant le locuteur et ses interactions au sein d'un groupe au centre de l'analyse, elle permet d'envisager le mélange des langues comme une pratique discursive naissant des échanges entre personnes, plutôt que comme le fait d'un hypothétique locuteur idéal. En prenant exemple sur une étude croisée menée sur deux corpus de conversation bilingue, nous montrons ainsi que cette méthodologie permet de dégager à la fois des tendances qui semblent valides indépendamment des groupes étudiés et des pratiques circonscrites à l'un ou l'autre. Nous en concluons que la recherche sur l'alternance codique ne peut faire l'économie d'analyses et de comparaisons de corpus, sous peine de transformer des idiosyncrasies en règles générales.

This article aims at demonstrating that corpus-based research is the methodology that is most apt at correctly describing the phenomena involved in code-switching. By putting the individual speakers and their interactions as a group at the center of the stage, it makes it possible to analyze code-switching as a speech-based phenomenon arising from interpersonal exchanges, rather than from a hypothetical ideal speaker. Basing my claims on the comparative analysis of two bilingual corpora, I will show that this methodology enables the linguist to isolate tendencies that seem valid independently of the groups under scrutiny, as well as phenomena that only appear in one group or the other. I conclude that research on code-switching, if it is to avoid mistaking idiosyncrasies for universals, should be carried out using corpus analyzes and systematic comparisons between corpora.

\section{INDEX}

Mots-clés : bilinguisme, alternance codique, analyse du discours, idiolectes, universaux du langage

Keywords : bilingualism, code-switching, discourse analysis, idiolects, language universals 


\section{AUTEUR}

\section{CHARLES BRASART}

Université Paris-Sorbonne, EA 3553 « Concepts \& Langages »

charles.brasart@paris-sorbonne.fr 